\title{
Growth factors and interleukin-6 across the lung circulation in pulmonary hypertension
}

\author{
N. Selimovic*, C-H. Bergh*, B. Andersson*, E. Sakiniene" \\ H. Carlsten ${ }^{\#}$ and B. Rundqvist*
}

ABSTRACT: The aim of our study was to assess the levels of growth factors and interleukin (IL)-6 across the pulmonary circulation in patients with pulmonary arterial hypertension (PAH) and correlate them with clinical and haemodynamic data and outcome.

Simultaneous arterial and pulmonary arterial blood samples in patients with PAH $(n=44)$ and controls $(n=20)$ were obtained during right heart catheterisation. Vascular endothelial growth factor (VEGF), platelet-derived growth factor (PDGF)-BB, transforming growth factor (TGF)- $\beta 1$ and IL-6 were measured using ELISA.

Arterial median (interquartile range) values for VEGF, PDGF-BB, TGF- $\beta 1$ and IL-6 were significantly higher in patients $\left(377(218-588)\right.$ versus $9.0 \mathrm{pg} \cdot \mathrm{mL}^{-1} ; 1,955(1,371-2,519)$ versus $306(131-502) \mathrm{pg} \cdot \mathrm{mL}^{-1} ; 26.42(11.3-41.1)$ versus $7.0(1.8-18.4) \mathrm{ng} \cdot \mathrm{mL}^{-1}$; and $3.98(0.7-8.1)$ versus $0.7 \mathrm{pg} \cdot \mathrm{mL}^{-1}$, respectively; $\mathbf{p}<\mathbf{0 . 0 0 1}$ for all variables). There was a consistent step-up of VEGF, PDGF-BB and TGF- $\beta 1$ across the lungs in PAH patients $(p<0.001, p=0.002$ and $p<0.001$, respectively), whereas in controls, arterial and pulmonary arterial serum levels of IL- 6 and growth factors were similar (statistically nonsignificant). In multivariate analysis, increased IL-6 levels predicted mortality (hazard ratio 1.08 (95\% confidence interval 1.02-1.15); $p=0.012$ ).

Our findings indicate increased release and/or decreased clearance of growth factors at the lung vascular level, which may contribute to vascular remodelling in PAH.

KEYWORDS: Growth substances, inflammation, pulmonary hypertension, remodelling

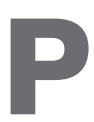

ulmonary arterial hypertension (PAH) is a progressive disease characterised by pulmonary vasculopathy leading to elevation in pulmonary vascular resistance, right heart failure and premature death [1]. Accumulated evidence indicates that cellular proliferation of endothelial cells, smooth muscle cells (SMCs) and fibroblasts plays an important role for the vascular remodelling characteristic of PAH [24]. Vascular cell proliferation is regulated by various growth factors and there is evidence indicating that growth signalling is altered for the vascular endothelial growth factor (VEGF), platelet-derived growth factor (PDGF) and transforming growth factor (TGF)- $\beta$ pathways in $\mathrm{PAH}$ [5-12]. These pathways may also represent new targets for pharmacological treatment [13-15].

VEGF is an endothelial cell-specific mitogen and potent angiogenic peptide secreted by a variety of cell types and tissues. VEGF and its receptors, Flt-1 and Flk-1, are expressed in the plexiform lesions, a unique vascular structure in PAH [16]. PDGF is a potent mitogen for fibroblasts and SMCs that promotes wound healing, whereas VEGF initiates angiogenesis and accelerates repair of the endothelial cell lining. In lung biopsies from patients with severe $\mathrm{PAH}$, the PDGF-AA chain was significantly increased [17] and pulmonary vascular expression of PDGF and the PDGF receptors was also found to be increased in explanted lungs from patients with end-stage PAH [14].

The TGF- $\beta$ superfamily includes TGF- $\beta$ isoforms (TGF- $\beta 1-5)$, bone morphogenetic proteins (BMPs), activins and inhibins. In pulmonary artery SMCs from patients with idiopathic PAH (IPAH), TGF$\beta 1$ causes enhanced cell proliferation, in contrast to the growth inhibitory effects observed in normal cells [12, 18]. TGF- $\beta 1$ enhances the deposition of extracellular matrix through promotion of synthesis and inhibition of the degradation.

The presence of lymphocytes adjacent to vascular lesions in patients with PAH and the association between PAH and connective tissue diseases suggest that immunological pathways also play a pathophysiological role $[5,8]$. Pro-inflammatory cytokines (interleukin (IL)-1 $\beta$, IL-6 and tumour necrosis factor- $\alpha$ ) may also contribute to the mechanisms leading to the proliferation of vascular cells [19].

\section{AFFILIATIONS}

Depts of ${ }^{*}$ Cardiology, and

\#Rheumatology, Sahlgrenska University Hospital, Gothenburg, Sweden.

\section{CORRESPONDENCE}

N. Selimovic

Dept of Cardiology

Sahlgrenska University Hospital

SE-413 45 Gothenburg

Sweden

E-mail: nedim.selimovic@

vgregion.se

Received:

Nov 182008

Accepted after revision:

March 162009

First published online:

March 262009 
Previous studies of growth factors in PAH have been carried out mainly on either animals or explanted lung tissue obtained from patients with end-stage disease during lung transplantation. The aim of the present study was to assess serum concentrations of VEGF, PDGF-BB, TGF- $\beta 1$ and IL- 6 across the pulmonary circulation in vivo by simultaneous arterial and pulmonary arterial (mixed venous) blood sampling in patients with PAH and to compare it with control subjects. In addition, serum levels of these growth factors and IL-6 were correlated with clinical and haemodynamic variables, and with outcome. Our hypothesis was that activation of these pathways could be reflected in an increased gradient of these mediators across the pulmonary vascular bed in patients with PAH.

\section{MATERIALS AND METHODS}

\section{Study population}

We prospectively studied 44 consecutive patients with pulmonary hypertension during diagnostic or follow-up right heart catheterisation. 43 patients fulfilled the diagnostic criteria for PAH according to the World Health Organization classification [20]. We included one patient with multiple peripheral pulmonary artery stenosis due to the clinical and haemodynamic similarities with PAH. Age, sex, aetiology, New York Heart Association (NYHA) class, 6-min walk test, current medication, basic laboratory tests, haemodynamics and outcome (alive or dead) were documented for all PAH patients. The control group comprised 20 patients with leftsided Wolff-Parkinson-White syndrome who were otherwise healthy (table 1). All control subjects underwent a clinical and echocardiographic study with normal results, and had normal routine blood tests. Our study was approved by the Institutional Review Board at the University of Gothenburg, Sweden, and all patients gave informed consent. The first patient was included in the study in February 2004. Follow-up was complete and outcome was determined for all patients by the end of the study, on December 31, 2007. The mean \pm SD follow-up time for PAH patients was $814 \pm 442$ days.
At the time of blood sampling for growth factors and IL-6, 25 patients were untreated with respect to specific PAH therapy. Among treated patients, six patients received the endothelin receptor antagonist bosentan, three patients received continuous i.v. epoprostenol, three patients received the phosphodiesterase type 5 inhibitor sildenafil, three patients received both prostacyclin and bosentan, two patients received bosentan and iloprost, and another two patients received a combination of bosentan, prostacyclin and sildenafil.

\section{Sample collection and assay}

Patients with $\mathrm{PAH}$ underwent standard right heart catheterisation using a Swan-Ganz catheter (7 F; Baxter Healthcare, Edwards Critical Care Division, Deerfield, IL, USA). The catheter was introduced using the Seldinger technique through the right internal jugular vein under fluoroscopic guidance. A left radial artery catheter was used to measure systemic arterial blood pressure and systemic arterial oxygen saturation and for blood sampling. The transpulmonary gradient was assessed by measuring the levels of the growth factors in blood samples taken simultaneously from the mixed venous blood of the pulmonary artery (venous samples) and radial artery (arterial samples).

Controls underwent radiofrequency ablation of the accessory left-sided pathway using transeptal puncture or a retrograde femoral artery approach. Blood samples from the pulmonary artery were obtained via a pigtail catheter introduced through the right femoral vein.

Blood samples were taken simultaneously from the pulmonary artery and radial artery $10 \mathrm{~min}$ after haemodynamic measurements were performed at rest in patients with PAH. In the controls, blood samples were collected simultaneously from the pulmonary artery and left atrium or femoral artery.

Serum was prepared by drawing $9 \mathrm{~mL}$ of blood in Venosafe $\mathrm{TM}^{\mathrm{TM}}$ tubes containing a clot activator (Terumo Europe NV, Leuven, Belgium), then allowing the tubes to stand for $60 \mathrm{~min}$ at $22^{\circ} \mathrm{C}$ to

\section{TABLE 1 Patient characteristics}

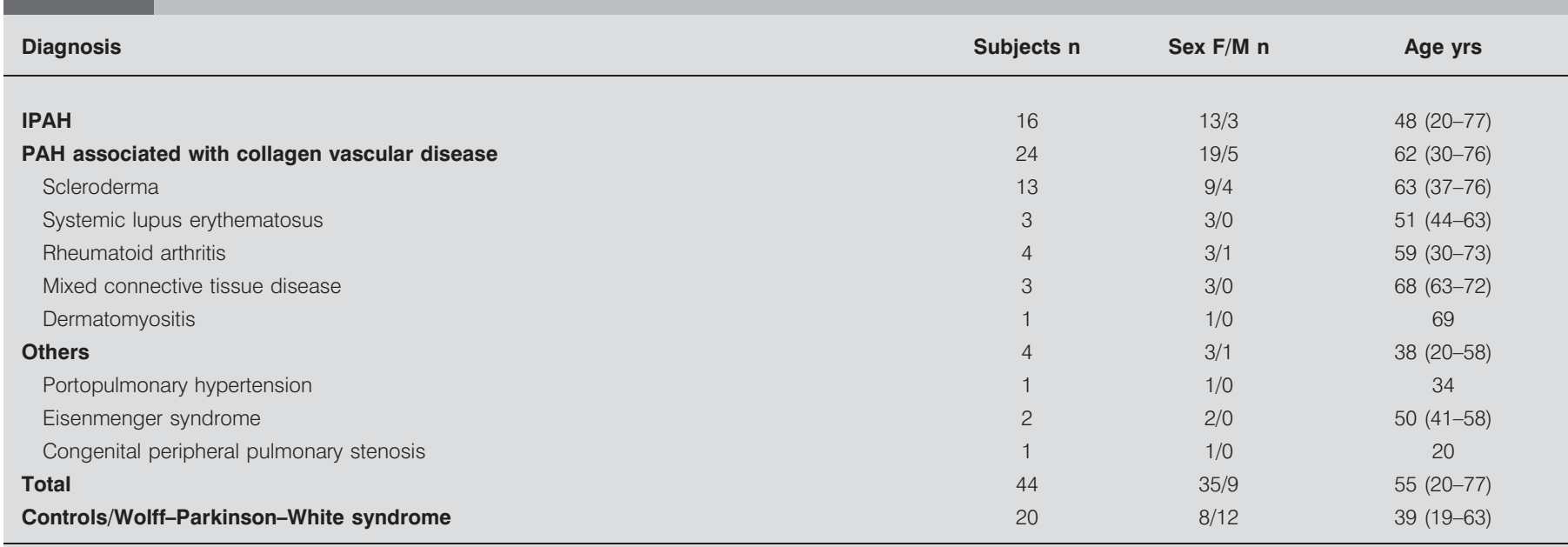

Data are presented as mean (range), unless otherwise indicated. F: female; M: male; IPAH: idiopathic pulmonary arterial hypertension; PAH: pulmonary arteria hypertension. 
ensure full clotting of serum. These samples were centrifuged shortly after clot formation. All samples were stored at $-70^{\circ} \mathrm{C}$ in aliquots and thawed only before measurement.

The levels of the VEGF, PDGF-BB, TGF- $\beta 1$ and IL-6 were assessed using an ELISA for these factors (Quantikine; R\&D Systems, Minneapolis, MN, USA). PDGF-BB is one of three isoforms: PDGF-AA, -AB and -BB. These three forms of PDGF have different but overlapping biological activities. A reason to assess the PDGF-BB dimer was the availability of a commercially and reliable human PDGF-BB immunoassay.

The minimum detectable levels of VEGF, PDGF-BB and TGF- $\beta 1$ were 9, 15 and $4.61 \mathrm{pg} \cdot \mathrm{mL}^{-1}$, respectively. The minimum detectable level of IL-6 was $<0.7 \mathrm{pg} \cdot \mathrm{mL}^{-1}$.

\section{Statistical analysis}

Data were entered in an electronic database and analysed using an SPSS program (version 15.0.1 for Windows; SPSS Inc., Chicago, IL, USA). Due to the skewed distribution of the growth factor and IL-6 levels, statistical analysis was performed using nonparametric methods and summary measures are presented as median (interquartile range). The differences between the levels of these variables among PAH patients versus controls were tested using the Mann-Whitney U-test. The differences between the levels of growth factors and IL-6 in radial artery versus pulmonary artery in the same group (PAH patients/controls) were tested using the Wilcoxon's signed-rank test.

The Spearman rank correlation was used to examine the correlation coefficient. Potential risk factors were initially analysed for significant association with mortality using the Cox proportional hazards model for continuous variables. Risk factors with a level of significance defined as $p<0.2$ in the univariate analysis were included in the multivariable model. A probability value of $\mathrm{p}<0.05$ was considered statistically significant.

\section{RESULTS}

\section{Study population}

The distribution of different forms of $\mathrm{PAH}$ and patient characteristics are shown in table 1. NYHA functional class, 6-min walking distance and haemodynamic variables of patients with PAH are presented in table 2. There were no significant differences regarding age between control subjects and patients with IPAH (mean \pm SD $39 \pm 16$ versus $48 \pm 18$ yrs; $\mathrm{p}=0.13$ ), but patients with $\mathrm{PAH}$ associated with collagen vascular diseases were older than controls (62 \pm 12 versus $39 \pm 16$ yrs; $\mathrm{p}<0.001)$.

\section{Growth factors and IL-6 across the lung circulation in patients with $P A H$}

Median serum arterial levels of VEGF, PDGF-BB, TGF- $\beta 1$ and IL-6 were significantly higher in patients with $\mathrm{PAH}$ compared with controls (377 (218-588) versus $9.0 \mathrm{pg} \cdot \mathrm{mL}^{-1} ; 1,955(1,371-$ $2,519)$ versus $306(131-502) \mathrm{pg} \cdot \mathrm{mL}^{-1} ; 26.42$ (11.3-41.1) versus 7.0 $(1.8-18.4) \mathrm{ng} \cdot \mathrm{mL}^{-1} ; 3.98(0.7-8.1)$ versus $0.7 \mathrm{pg} \cdot \mathrm{mL}^{-1}$, respectively). Similarly, the median serum levels of VEGF, PDGF-BB, TGF- $\beta 1$ and IL-6 in venous blood samples were significantly higher in PAH patients compared with controls (169 (100-277) versus $9.0 \mathrm{pg} \cdot \mathrm{mL}^{-1} ; 1,263(733-1,967)$ versus $217(138-448) \mathrm{pg} \cdot \mathrm{mL}^{-1} ; 14.7$

(8.5-33.3) versus $5.9(2.6-12.5) \mathrm{ng} \cdot \mathrm{mL}^{-1} ; 4.19$ (1.6-11.9) versus $0.7 \mathrm{pg} \cdot \mathrm{mL}^{-1}$, respectively; fig. $\left.1 \mathrm{a}-\mathrm{d}\right)$.

There was no influence of age or sex on the levels of growth factors and IL-6 among patients or in the control group (data not shown).

There was a significant difference between the arterial and venous blood levels (transpulmonary gradient) of all growth factors in patients with $\mathrm{PAH}$, whereas there were no significant differences between levels of IL-6 or growth factors in the pulmonary artery versus the radial artery/left atrium in control subjects (fig. 1a-d).

When the group of patients with IPAH was compared with the group of patients with collagen vascular diseases, no significant differences were observed regarding arterial and mixed venous levels of VEGF, PDGF-BB, TGF- $\beta 1$ and IL-6 (table 3).

Patients with other forms of PAH had similar levels of growth factors to the other groups of PAH in both mixed venous and arterial samples.

There were no significant differences in transpulmonary gradient of growth factors between these two groups of patients. Patients without treatment $(n=25)$ showed significant increases in levels of growth factors and IL-6 in pulmonary arterial and arterial samples, and the transpulmonary gradients of growth factors as compared with controls (data not shown).

There was no significant correlation between the levels of growth factors, levels of IL-6, and clinical and haemodynamic variables reflecting the severity of pulmonary hypertension. TGF- $\beta 1$ correlated significantly with PDGF-BB in the pulmonary artery $(\mathrm{r}=0.58 ; \mathrm{p}<0.001)$, but not in the systemic circulation $(\mathrm{r}=0.27 ; \mathrm{p}=0.07)$. A weak correlation was found between VEGF and PDGF-BB levels in the pulmonary artery $(r=0.36$; $\mathrm{p}=0.017)$.

TABLE 2 Clinical and haemodynamic characteristics of patients with pulmonary arterial hypertension

Characteristic

Subjects $\mathbf{n}$

NYHA functional class I/II/III/IV $\mathbf{n}$ 6MWD $\mathrm{m}$

RAP $\mathrm{mmHg}$

$\bar{P}$ pa $\mathbf{m m H g}$

Ppcw mmHg

$\mathrm{Sa}, \mathrm{O}_{2} \%$

Cardiac index $\mathrm{L} \cdot \mathrm{min}^{-1} \cdot \mathrm{m}^{-2}$

PVR WU
Value

44
$2 / 4 / 35 / 3$
$342(211-422)$
$5(3-9)$
$44(33-56)$
$7(6-11)$
$93.9(90.3-97.2)$
$2.99(2.7-3.3)$
$7.2(4.5-12.3)$

Data are presented as median (range), unless otherwise indicated. NYHA: New York Heart Association; 6MWD: 6-min walking distance; RAP: right atrial pressure; $\bar{P}_{\text {pa: }}$ mean pulmonary artery pressure; Ppcw: pulmonary capillary wedge pressure; $\mathrm{Sa}, \mathrm{O}_{2}$ : arterial oxygen saturation; PVR: pulmonary vascular resistance; WU: Wood units. 

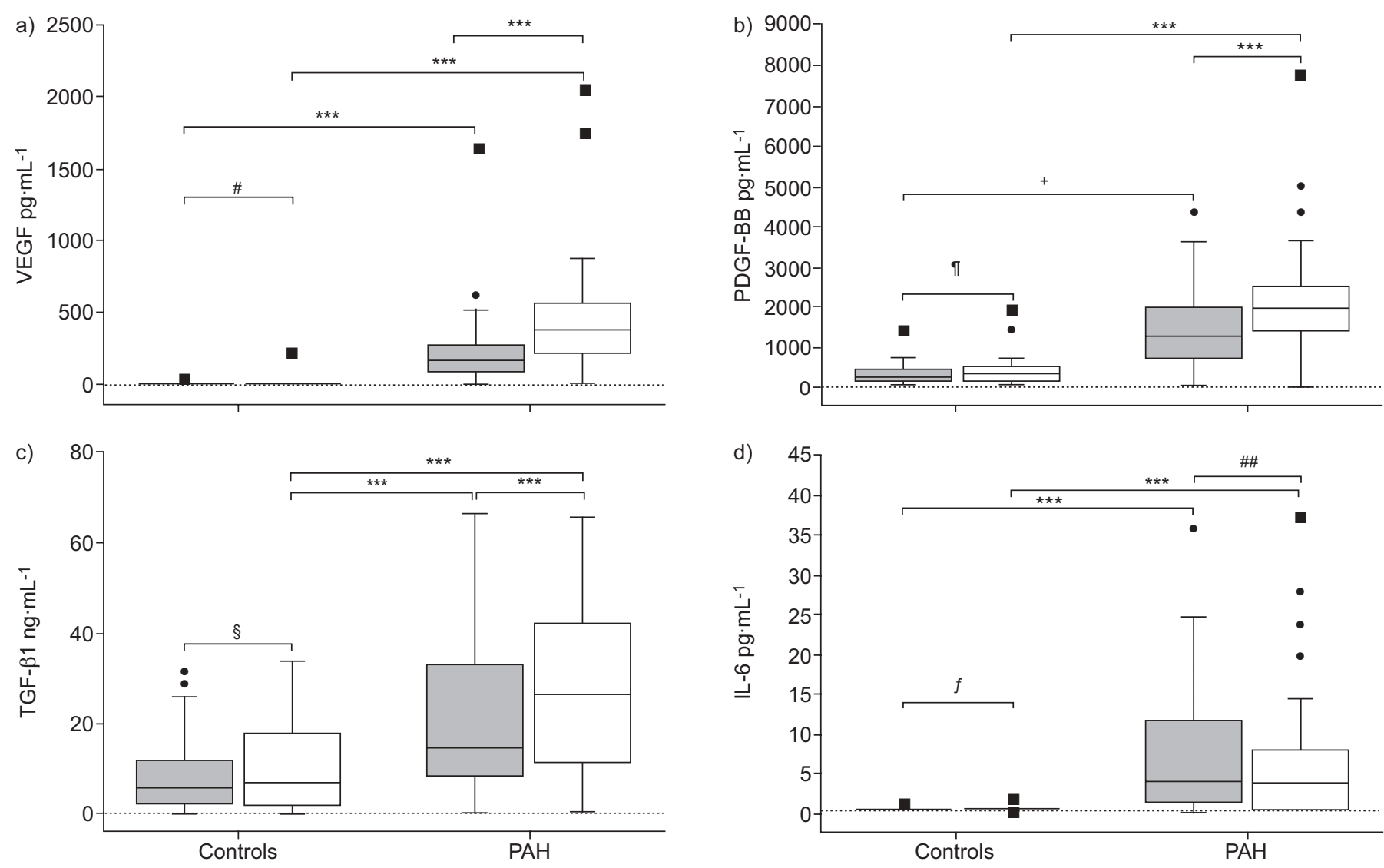

FIGURE 1. Serum levels of a) vascular endothelial growth factor (VEGF), b) platelet-derived growth factor (PDGF)-BB, c) transforming growth factor (TGF)- $\beta 1$ and d) interleukin (IL)-6 in control subjects $(n=20)$ and in patients with pulmonary arterial hypertension (PAH; $n=44)$. $\square$ : radial artery/left atrium; 1 : pulmonary artery; $\bullet$ : outlier extending $>1.5$ box-lengths from the edge of the box; $\mathbf{\square}$ : extreme outlier extending $>3$ box-lengths from the edge of the box. ${ }^{*}: p=0.36 ;{ }^{*}: p=0.384 ;{ }^{+}: p=0.002 ;{ }^{s}: p=0.38$; f: $p=0.86 ;{ }^{\# \#: ~} p=0.14$. ${ }^{* *}: p<0.001$.

\section{Association between the levels of growth factors and IL-6 with outcome}

The 15 patients that died during follow-up had a tendency to lower arterial VEGF serum levels than those who survived ( $311 \pm 48$ versus $\left.525 \pm 85 \mathrm{pg} \cdot \mathrm{mL}^{-1} ; \mathrm{p}=0.09\right)$. There were no significant differences between survivors and nonsurvivors regarding the 6-min walk test, mean pulmonary arterial pressure and pulmonary vascular resistance, except for age. The results of the univariate analysis of potential risk factors associated with mortality during this period are shown in table 4 . The result of the multivariate analysis is presented in table 5. Elevated serum levels of IL-6 emerged as an independent risk factor for mortality during the observation period.

\section{DISCUSSION}

The present study showed that serum levels of VEGF, PDGF-BB, TGF- $\beta 1$ and IL-6 were significantly higher in patients with PAH compared with controls. Furthermore, there was a consistent step-up of growth factors across the pulmonary vasculature in $\mathrm{PAH}$ patients that was not found in the control group. The higher arterial serum concentrations of growth factors versus mixed venous levels indicate increased production and release of these mediators within the pulmonary vasculature. A decreased pulmonary clearance of these substances may also contribute to the increased transpulmonary gradients. Both arterial and mixed venous levels and the transpulmonary gradients of growth factors were similar among patients with IPAH and in patients with PAH associated with connective tissue diseases. This indicates that the pulmonary vasculopathy is also a source of the high serum levels of growth factors in associated PAH. A concomitant increase in all three growth factors in patients with PAH suggests common stimuli for an increase in their production and/or decreased clearance. However, we did not find any significant relationship between the levels of VEGF, PDGF-BB and TGF- $\beta 1$ with clinical or haemodynamic variables, such as pulmonary artery pressures or pulmonary vascular resistance reflecting the severity of PAH.

In the present study, elevated arterial IL-6 concentrations were independently associated with mortality in the multivariate analysis, suggesting that IL-6 may be a biomarker for the prognosis of PAH. There were significantly higher concentrations of IL-6 in both arterial and mixed venous blood from patients with $\mathrm{PAH}$ compared with controls. However, there was no step-up of IL-6 across the lungs in either of the study groups, suggesting that systemic levels of IL-6 are not determined by the lungs in PAH.

VEGF is strongly expressed in the plexiform lesions in the lungs from patients with severe IPAH and secondary forms of PAH [16]. Experimental studies have shown that chronic 


\begin{tabular}{|c|c|c|c|c|}
\hline \multirow{2}{*}{$\begin{array}{l}\text { TABLE } 3 \\
\text { Variables }\end{array}$} & \multicolumn{4}{|c|}{$\begin{array}{l}\text { Interleukin (IL)- } 6 \text { and growth factor levels in } \\
\text { patients with idiopathic pulmonary arterial } \\
\text { hypertension (IPAH) or pulmonary arterial } \\
\text { hypertension (PAH) associated with collagen } \\
\text { vascular diseases (CVD) }\end{array}$} \\
\hline & & IPAH patients & $\begin{array}{l}\text { PAH associated } \\
\text { with CVD }\end{array}$ & p-value \\
\hline \multicolumn{2}{|l|}{ Sul } & 16 & 24 & \\
\hline \multicolumn{2}{|c|}{ IL-6 RA pg $\cdot \mathrm{mL}^{-1}$} & $4.3(1.3-7.4)$ & $3.3(0.7-11.1)$ & 0.744 \\
\hline \multicolumn{2}{|c|}{ IL-6 PA pg $\cdot \mathrm{mL}^{-1}$} & $4.1(1.4-8.5)$ & $4.5(1.2-16.9)$ & 0.391 \\
\hline \multicolumn{2}{|c|}{ VEGF RA pg.mL $\mathrm{mL}^{-1}$} & $397(141-679)$ & $377(247-502)$ & 0.868 \\
\hline \multicolumn{2}{|c|}{ VEGF PA pg $\cdot \mathrm{mL}^{-1}$} & $176(52-343)$ & 159 (113-208) & 0.814 \\
\hline \multicolumn{2}{|c|}{ PDGF-BB RA pg.mL $\mathrm{mL}^{-1}$} & 1634 (1353-2150) & $2004(1385-2608)$ & 0.44 \\
\hline \multicolumn{2}{|c|}{ PDGF-BB PA pg.mL $\mathrm{mL}^{-1}$} & 1440 (954-1952) & 977 (603-1713) & 0.172 \\
\hline \multicolumn{2}{|c|}{$\mathrm{TGF}-\beta 1 \mathrm{RA} \mathrm{ng} \cdot \mathrm{mL}^{-1}$} & $21.4(11-42.3)$ & $29.4(12-39)$ & 0.619 \\
\hline \multicolumn{2}{|c|}{ TGF- $\beta 1$ PA $n g \cdot \mathrm{mL}^{-1}$} & $14.8(7.4-37.3)$ & $14.7(8.3-32.2)$ & 0.868 \\
\hline
\end{tabular}

Data are presented as median (interquartile range), unless otherwise indicated. RA: radial artery; PA: pulmonary artery; VEGF: vascular endothelial growth factor; PDGF: platelet-derived growth factor; TGF: transforming growth factor.

hypoxia increases lung tissue VEGF expression and that VEGF is a modulator of chronic hypoxia-induced pulmonary vascular remodelling and may also provide protection from disease progression [21]. In the current study, we did not find any correlation between VEGF and arterial or mixed venous oxygen saturation. Our results regarding circulating levels of VEGF agree with the findings in a previous report of elevated serum VEGF levels in peripheral venous blood samples from patients with PAH [22]. In contrast, BENISTY et al. [23] showed no significant differences in circulating VEGF levels in peripheral venous blood samples between patients and control subjects. In their study, BENISTY et al. [23] measured plasma VEGF, and this may have resulted in an underestimation of circulating VEGF levels, as VEGF can be stored in platelets. Intravenous epoprostenol therapy may increase the levels of VEGF in serum and platelet lysate [22]. However, only a small number of subjects in the present study were receiving epoprostenol, hence this confounder cannot explain the increase in VEGF and, importantly, patients without treatment showed significant increases in both arterial and mixed venous VEGF levels.

Patients with PAH in the current study had a four to five times higher serum level of PDGF-BB with a significant transpulmonary gradient compared with control subjects. This finding is consistent with a recent report that demonstrated increased expression of PDGF in explanted lungs obtained from $\mathrm{PAH}$ patients compared with donor lung tissue [14]. The same investigators also showed that increased PDGF expression was localised to SMCs and endothelial cells in small remodelled pulmonary arteries. Conversely, another investigation observed that circulating PDGF was slightly decreased in patients with $\mathrm{PAH}$ compared with control subjects [22]. In their study, EDDAHIBI et al. [22] took blood samples from peripheral veins, which may not reflect the pulmonary circulation. This makes their findings difficult to compare with our results.

\section{TABLE 4 Univariate analysis of possible predictors of mortality in patients with pulmonary arterial hypertension}

\begin{tabular}{|c|c|c|}
\hline Variable & HR $(95 \% \mathrm{Cl})$ & p-value \\
\hline IL-6 RA pg $\cdot \mathrm{mL}^{-1}$ & $1.07(1.02-1.13)$ & 0.012 \\
\hline VEGF RA pg. $\mathrm{mL}^{-1}$ & $0.99(0.99-1.0)$ & 0.06 \\
\hline PDGF-BB RA pg. $\mathrm{mL}^{-1}$ & $1.0(0.99-1.0)$ & 0.12 \\
\hline TGF- $\beta 1$ RA $n g \cdot \mathrm{mL}^{-1}$ & $1.0(0.97-1.03)$ & 0.84 \\
\hline Age yrs & $1.05(1.01-1.09)$ & 0.026 \\
\hline PVR WU & $1.03(0.93-1.13)$ & 0.586 \\
\hline 6MWD m & $0.997(0.99-1.01)$ & 0.128 \\
\hline $\mathrm{Sa}, \mathrm{O}_{2} \%$ & $0.94(0.879-1.01)$ & 0.11 \\
\hline
\end{tabular}

HR: hazard ratio; IL: interleukin; RA: radial artery; VEGF: vascular endothelial growth factor; PDGF: platelet-derived growth factor; TGF: transforming growth factor; PVR: pulmonary vascular resistance; WU: Wood units; 6MWD: 6-min walk distance; $\mathrm{Sa}_{1} \mathrm{O}_{2}$ : arterial oxygen saturation.

In an animal model, the PDGF receptor antagonist (STI571imatinib mesylate) was recently shown to reverse pulmonary hypertension [13]. In small clinical studies, treatment with the PDGF receptor antagonist imatinib had beneficial effects in patients with advanced PAH. This suggests that PDGF may have an important pathophysiological role in PAH [15].

There is growing evidence that abnormalities of TGF- $\beta$ superfamily signalling are linked to the pathogenesis of severe PAH [11, 24]. In the present study, we found significantly higher levels of TGF- $\beta 1$ in PAH patients in comparison with control subjects. Failure of the growth inhibitory effects of BMPs could contribute to the pulmonary vascular remodelling in PAH. There is evidence that TGF- $\beta 1$ and BMPs inhibit the serum-stimulated proliferation of pulmonary artery SMCs (PASMCs) from normal subjects and patients with secondary PAH (Eisenmenger patients). In contrast, TGF- $\beta 1$ enhanced the serum-stimulated proliferation of PASMCs from IPAH patients [12]. This raises additional questions regarding the interactions of the TGF- $\beta$ /BMP pathways with other pathways known to be involved in the regulation of PASMC growth.

We observed significant correlation between circulating levels of TGF- $\beta 1$ and PDGF-BB, and VEGF and PDGF-BB, consistent with previously demonstrated interactions and correlations

\begin{tabular}{lcc} 
TABLE 5 & $\begin{array}{l}\text { Multivariate predictors of mortality in patients with } \\
\text { pulmonary arterial hypertension }\end{array}$ \\
Variable & HR $\mathbf{( 9 5 \% ~ C l )}$ & p-value \\
\hline IL-6 RA pg $\cdot \mathbf{~ m L}^{-1}$ & $1.08(1.02-1.15)$ & 0.012 \\
VEGF RA pg $\mathbf{~ m L}^{-1}$ & $0.99(0.99-1.01)$ & 0.152 \\
PDGF-BB RA $\mathbf{~ g ~} \cdot \mathbf{m L}^{-1}$ & $1.0(0.99-1.0)$ & 0.285 \\
Age $\mathbf{y r s}$ & $1.03(0.98-1.09)$ & 0.207 \\
\hline
\end{tabular}

HR: hazard ratio; IL: interleukin; RA: radial artery; VEGF: vascular endothelial growth factor; PDGF: platelet-derived growth factor 
between these growth factors. This suggests common stimuli regulating the release of growth factors in PAH [22, 23, 25, 26].

Patients with PAH associated with connective tissue diseases had a tendency towards higher levels of circulating IL-6 compared with patients with IPAH, but the differences were not statistically significant. Similar findings of increased IL-6 serum concentrations in patients with severe $\mathrm{PAH}$ were reported by others [19]. In contrast, HOEPER and WeLTE [27] did not find any significant increase of serum IL-6 in patients with PAH. In their study, HOEPER and WELTE [27] obtained blood samples from a peripheral vein, which makes their result difficult to compare with the findings in the present study. In addition, from the available data, it is not possible to clarify whether the various study populations were comparable.

IL-6, a pro-inflammatory and vasodepressor cytokine, showed a significant association with mortality in patients with PAH. Similar findings have been reported in patients with congestive heart failure [28] and in patients with acute coronary syndromes [29]. IL-6 is produced not only by leukocytes [30], but also by the vascular tissue, both endothelial and SMCs, and myocytes and fibroblasts in various organs, including the lung. These data suggest that IL-6, as a systemic mediator of immune and inflammatory response [30], has a role in the pathophysiology of different cardiovascular diseases, such as acute coronary syndromes, congestive heart failure and pulmonary hypertension [28, 29].

\section{Study limitations}

The sample size in the present study is small with respect to survival analysis. Therefore, the predictive value of IL-6 for mortality in patients with $\mathrm{PAH}$ should be interpreted with caution. Another limitation of our study is that arterial blood samples were taken from either the left atrium or alternatively the femoral artery in control subjects, but from the radial artery in PAH patients. However, blood from the radial artery/ femoral artery should be a reliable substitute for an aortic sample because of the rapid transient time and low surface area of the large arterial tree. In addition, pulmonary blood flow was not measured in the control group, which hampers estimation of a potential flow-dependent washout of growth factors and IL- 6 from the pulmonary vasculature. However, no correlation between the levels of growth factors and cardiac output was found in PAH patients.

In summary, our study has demonstrated that serum levels of the growth factors VEGF, PDGF-BB and TGF- $\beta 1$, and IL- 6 were significantly higher in both systemic and mixed venous samples from patients with PAH compared with control subjects. Furthermore, there was a significant step-up of all growth factors across the lungs in PAH patients. Importantly, similar results were obtained in both IPAH and associated $\mathrm{PAH}$, as well as in de novo $\mathrm{PAH}$ patients without treatment. These findings indicate an increased release and/or decreased clearance of these mediators at the lung vascular level. IL-6 serum levels were higher among PAH patients and IL-6 was an independent predictor of adverse outcome. With a shift from focus on vasoconstriction to abnormal vascular cellular proliferation in the small vessels of the lungs in clinical PAH, the findings in the present study support the hypothesis that growth factors may contribute to vascular remodelling in $\mathrm{PAH}$.

\section{STATEMENT OF INTEREST}

None declared.

\section{ACKNOWLEDGEMENTS}

The authors would like to thank L. Bergfeldt and S. Gizurarson (both from Sahlgrenska University Hospital, Gothenburg, Sweden) for their help in recruiting subjects to form the control group. We also thank M. Erlandsson (Dept of Rheumatology and Inflammation Research, Sahlgrenska Academy, University of Gothenburg) for help with immunoassay analysis and E. Angwald (Wallenberg Laboratory for Cardiovascular Research, Sahlgrenska University Hospital) for collection and preparation of blood samples.

\section{REFERENCES}

1 Rubin LJ. Primary pulmonary hypertension. N Engl J Med 1997; 336: 111-117.

2 Meyrick B, Reid L. Hypoxia-induced structural changes in the media and adventitia of the rat hilar pulmonary artery and their regression. Am J Pathol 1980; 100: 151-178.

3 Pietra GG, Edwards WD, Kay JM, et al. Histopathology of primary pulmonary hypertension. A qualitative and quantitative study of pulmonary blood vessels from 58 patients in the National Heart, Lung, and Blood Institute, Primary Pulmonary Hypertension Registry. Circulation 1989; 80: 1198-1206.

4 Farber HW, Loscalzo J. Pulmonary arterial hypertension. N Engl J Med 2004; 351: 1655-1665.

5 Tuder RM, Groves B, Badesch DB, et al. Exuberant endothelial cell growth and elements of inflammation are present in plexiform lesions of pulmonary hypertension. Am J Pathol 1994; 144: 275-285.

6 Tuder RM, Flook BE, Voelkel NF. Increased gene expression for VEGF and the VEGF receptors KDR/Flk and Flt in lungs exposed to acute or to chronic hypoxia. Modulation of gene expression by nitric oxide. J Clin Invest 1995; 95: 1798-1807.

7 Voelkel NF, Tuder RM. Cellular and molecular mechanisms in the pathogenesis of severe pulmonary hypertension. Eur Respir J 1995 8: 2129-2138.

8 Voelkel NF, Cool C, Lee SD, et al. Primary pulmonary hypertension between inflammation and cancer. Chest 1998; 114: Suppl. 3, 225S-230S.

9 Voelkel NF, Vandivier RW, Tuder RM. Vascular endothelial growth factor in the lung. Am J Physiol Lung Cell Mol Physiol 2006; 290: L209-L221.

10 Voelkel NF, Douglas IS, Nicolls M. Angiogenesis in chronic lung disease. Chest 2007; 131: 874-879.

11 Richter A, Yeager ME, Zaiman A, et al. Impaired transforming growth factor-beta signaling in idiopathic pulmonary arterial hypertension. Am J Respir Crit Care Med 2004; 170: 1340-1348.

12 Morrell NW, Yang X, Upton PD, et al. Altered growth responses of pulmonary artery smooth muscle cells from patients with primary pulmonary hypertension to transforming growth factor- $\beta 1$ and bone morphogenetic proteins. Circulation 2001; 104: 790-795.

13 Schermuly RT, Dony E, Ghofrani HA, et al. Reversal of experimental pulmonary hypertension by PDGF inhibition. J Clin Invest 2005; 115: 2811-2821.

14 Perros F, Montani D, Dorfmuller P, et al. Platelet-derived growth factor expression and function in idiopathic pulmonary arterial hypertension. Am J Respir Crit Care Med 2008; 178: 81-88.

15 Ghofrani HA, Seeger W, Grimminger F. Imatinib for the treatment of pulmonary arterial hypertension. $N$ Engl J Med 2005; 353: 1412-1413.

16 Hirose S, Hosoda Y, Furuya S, et al. Expression of vascular endothelial growth factor and its receptors correlates closely with formation of the plexiform lesion in human pulmonary hypertension. Pathol Int 2000; 50: 472-479. 
17 Humbert M, Monti G, Fartoukh M, et al. Platelet-derived growth factor expression in primary pulmonary hypertension: comparison of HIV seropositive and HIV seronegative patients. Eur Respir J 1998; 11: 554-559.

18 Du L, Sullivan CC, Chu D, et al. Signaling molecules in nonfamilial pulmonary hypertension. N Engl J Med 2003; 348: 500-509.

19 Humbert M, Monti G, Brenot F, et al. Increased interleukin-1 and interleukin-6 serum concentrations in severe primary pulmonary hypertension. Am J Respir Crit Care Med 1995; 151: 1628-1631.

20 Simonneau G, Galie N, Rubin LJ, et al. Clinical classification of pulmonary hypertension. J Am Coll Cardiol 2004; 43: Suppl. 12, 5S-12S.

21 Partovian C, Adnot S, Raffestin B, et al. Adenovirus-mediated lung vascular endothelial growth factor overexpression protects against hypoxic pulmonary hypertension in rats. Am J Respir Cell Mol Biol 2000; 23: 762-771.

22 Eddahibi S, Humbert M, Sediame S, et al. Imbalance between platelet vascular endothelial growth factor and platelet-derived growth factor in pulmonary hypertension. Effect of prostacyclin therapy. Am J Respir Crit Care Med 2000; 162: 1493-1499.

23 Benisty JI, McLaughlin VV, Landzberg MJ, et al. Elevated basic fibroblast growth factor levels in patients with pulmonary arterial hypertension: matrix metalloproteinases in the urine of patients with pulmonary arterial hypertension. Chest 2004; 126: $1255-1261$.

24 Tuder RM, Yeager ME, Geraci M, et al. Severe pulmonary hypertension after the discovery of the familial primary pulmonary hypertension gene. Eur Respir J 2001; 17: 1065-1069.

25 Cheng D, Lee YC, Rogers JT, et al. Vascular endothelial growth factor level correlates with transforming growth factor- $\beta$ isoform levels in pleural effusions. Chest 2000; 118: 1747-1753.

26 Pollman MJ, Naumovski L, Gibbons GH. Vascular cell apoptosis: cell type-specific modulation by transforming growth factor- $\beta 1$ in endothelial cells versus smooth muscle cells. Circulation 1999; 99: 2019-2026.

27 Hoeper MM, Welte T. Systemic inflammation, COPD, and pulmonary hypertension. Chest 2007; 131: 634-635.

28 Deswal A, Petersen NJ, Feldman AM, et al. Cytokines and cytokine receptors in advanced heart failure: an analysis of the cytokine database from the Vesnarinone trial (VEST). Circulation 2001; 103: 2055-2059.

29 Hartford M, Wiklund O, Mattsson Hulten L, et al. C-reactive protein, interleukin-6, secretory phospholipase A2 group IIA and intercellular adhesion molecule- 1 in the prediction of late outcome events after acute coronary syndromes. J Intern Med 2007; 262: 526-536.

30 Kishimoto T. The biology of interleukin-6. Blood 1989; 74: 1-10. 